\title{
Caracterização da fauna edáfica na produção pecuária em distintos sistemas de pastoreio
}

\section{Characterization of edaphic fauna in livestock production in different systems of grazing}

\author{
Silvia Carambula ${ }^{1}$, Ruben $\mathrm{Cruz}^{2}$, Juan Roberto Dutra ${ }^{3}$, Leonardo de Melo Menezes*1, Marco \\ Antonio Benamú ${ }^{2}$
}

\begin{abstract}
RESUMO
Este trabalho teve o objetivo de avaliar a densidade e diversidade da faunaa edáfica em dois períodos do ano (inverno e primavera) em sistema de pastoreio contínuo racional Voisin (PRV), com três profundidades do solo. As avaliações foram realizadas conforme o método recomendado pelo programa "Tropical soil Biology and Fertility" (TSBF). A maior densidade da fauna edáfica foi identificada no PRV durante o período de primavera, apesar de termos uma densidade mais baixa de insetos comparado com o inverno. Foi observada a similaridade da fauna nas diferentes profundidades do solo, onde se observou que a profundidade de $0 \mathrm{a} 10 \mathrm{~cm}$ e muito similar à de $10 \mathrm{a} 20 \mathrm{~cm}$ em o tratamento com exceção do período de PRV inverno, onde a fauna encontrada na cobertura e nos estratos de 0 a $10 \mathrm{~cm}$ são mais semelhantes considerando a diversidade. Não foi encontrada diferença significativa enquanto a função biológica da fauna edáfica e o tipo de manejo de pastoreio ou a estação do ano que foram coletadas as amostras. O maior número de indivíduos por família maior foi encontrado no manejo PRV durante a estação de inverno, no entanto a diversidade entre estes indivíduos foi mais baixa que nos demais tratamentos.
\end{abstract}

Palavras-chave: biologia do solo; campo natural; ecologia do pastejo; entomologia; pastejo rotativo;

\begin{abstract}
The objective of this work was to evaluate the density and diversity of the edaphic fauna in two periods of the year (winter and spring) in a Voisin rational continuous grazing system (PRV), with three soil depths. The evaluations were carried out according to the method recommended by the "Tropical soil Biology and Fertility" (TSBF) program. The highest density of edaphic fauna was identified in the PRV during the spring period, despite having a lower density of insects compared to winter. The similarity of the fauna in the different depths of the soil was observed, where it was observed that the depth of 0 to $10 \mathrm{~cm}$ is very similar to that of 10 to $20 \mathrm{~cm}$ in the treatment, with the exception of the winter PRV period, where the fauna found in the cover and in the strata from 0 to $10 \mathrm{~cm}$ are more similar considering the diversity. No significant difference was found regarding the biological function of the edaphic fauna and the type of grazing management or the season in which the samples were collected. The largest number of individuals per larger family was found in the PRV management during the winter season, however the diversity among these individuals was lower than in the other treatments.
\end{abstract}

Keywords: entomology; grazing ecology; natural field; rotary grazing; soil biology; 


\section{INTRODUÇÃO}

O crescente interesse por novas formas de produzir alimentos aliado à possibilidade da recuperação e conservação do potencial produtivo e saúde do solo nos desafiam a conhecer a composição e ecologia da fauna edáfica, bem como a possibilidade do uso deste conhecimento como ferramenta indicadora de qualidade do solo. O comportamento de sua riqueza taxonômica, diversidade, densidade, biomassa e composição funcional pode ser utilizado diretamente na avaliação de diferentes usos e manejos do solo (Villalobos et al., 2000; Marín, Feijoo y Peña, 2001; Zerbino, 2005; Zerbino et al., 2008; Huerta-Lwanga et al., 2008).

A fauna edáfica está constituída por uma grande variedade de invertebrados que realizam uma ou várias etapas de sua vida em diversas profundidades do perfil do solo (Anderson, 1988) e podem ser classificados em relação a sua taxonomia, diâmetro corporal, hábito de alimentação, entre outras. Tem importante atuação nos processos de decomposição, mineralização e umidificação de resíduos orgânicos; imobilização e mobilização de macro e micronutrientes; fixação de nitrogênio atmosférico; estruturação e agregação do solo e consequente conservação e regulação de pragas e doenças (autoregulação), beneficiando os sistemas de produção como um todo.

A macrofauna do solo inclui mais de 20 grupos taxonômicos (minhocas, cupins, formigas, centopéias, tesourinhas, grilos, baratas etc.), com dimensões acima de $10 \mathrm{~mm}$ de comprimento e $2 \mathrm{~mm}$ de diâmetro. Os organismos que compõem a macrofauna do solo são denominados "engenheiros do ecossistema", pois influem na disponibilidade de recursos para outros organismos, escavam, ingerem e transportam material mineral e orgânico do solo, habitando os espaços porosos do solo sendo afetados pela compactação de este (HEISLER \& KAISER, 1995; CRUZ \& BENAMU, 2015) participando indiretamente a regulação da decomposição de matérias orgânicas do solo (MOS).

A mesofauna inclui animais de $100 \mu \mathrm{m}-2 \mathrm{~mm}$ que habitam os espaços porosos do solo e não são capazes de criar galerias próprias. São exemplos: colêmbolos, dipluras, proturas e enquitreídeos, que se alimentam da matéria orgânica, de animais menores (nematóides) e de microrganismos. 
Dentre os métodos de pastejo mais comumente encontrados em sistemas extensivos destacam-se o Pastoreio Racional Voisin (PRV), método de pastoreio rotativo que leva em consideração o manejo do complexo solo-planta-animal, onde a intervenção do homem se dá através da subdivisão da área em piquetes; neste sentido, busca-se o direcionamento do gado para aqueles que apresentam o pasto no seu tempo de repouso adequado (Berton et al., 2011). Em outro lado, o pastoreio contínuo, no qual o gado fica sobre uma mesma área de pastagem durante um período indefinido (Melado, 2000).

O presente trabalho buscou investigar e caracterizar a fauna edáfica de dois sistemas de pastejo (contínuo e rotativo) em duas épocas do ano (primavera e inverno), em uma área localizada no Bioma Pampa.

\section{MATERIAL E MÉTODOS}

\section{Descrições da área experimental}

A pesquisa foi realizada em duas propriedades particulares caraterizadas pela utilização exclusiva de mão de obra familiar. A primeira utiliza o Pastoreio Racional Voisin desde o ano de 2012. Esta propriedade está dividida em 77 potreiros com áreas em 54 hectares; a propriedade 2 é lindeira com a propriedade 1 (campos contíguos), dedicada a pecuária extensiva na mesma localidade, sem deferimento de campo. As duas propriedades estão localizadas no interior do Uruguai, mais precisamente na divisa entre os Departamentos de Rivera e Tacuarembó, em uma região denominada de Laureles, coordenadas geográficas: $-31.323600,-55.977226$.

Os solos são superficiais de basalto. Os solos superficiais se classificam como Litossolos. Este grupo apresenta frequentemente vegetação natural de árvores e arbustos. Integra as unidades da Coxilha de Haedo-Paso de los Toros e Três Cerros, a escala 1:1.000.000 (D.S.F.). O clima da região corresponde ao tipo CFA de acordo com a classificação de Koppen, com características de cálido e úmido, com chuvas durante todo o ano e a temperatura do mês mais quente acima de 22 graus. A precipitação média anual do Uruguai é de 1300 mm, o que estabelece uma condição de clima chuvoso, com estação seca definida, com alta variabilidade interanual. 


\section{Procedimento Metodológico}

O delineamento experimental adotado foi de blocos ao acaso, com cinco repetições, três profundidades de amostragem de solo (cobertura, 0 a $10 \mathrm{~cm}$ e 10 a $20 \mathrm{~cm}$ ) e duas épocas de amostragem - inverno (julho de 2018) e primavera (novembro de 2018).

Como método de coleta de amostras foram seguidas as recomendações do TSBF (Tropical Soil Biology and Fertility) modificado (Anderson \& Ingram,1993) e todos os tratamentos se realizaram em transeptos de 25 metros, localizados em forma central dentro do tratamento. Os pontos de coleta foram georeferenciados, para permitir sua réplica.

Todas as amostras obtidas com os diferentes métodos de amostragem foram devidamente acondicionadas e rotuladas, e posteriormente transladados ao Laboratório de Eco toxicologia em Artrópodes Terrestres do Centro Universitário de Rivera (CUR). No laboratório as amostras passaram pelos seguintes procedimentos:

Extração manual: As amostras de cobertura foram retiradas da serapilheira e as amostras do solo localizado na projeção da sonda metálica $(25$ x $25 \mathrm{~cm})$. As amostras de solo foram separadas em camadas de $0-10 \mathrm{~cm}$ e $10-20 \mathrm{~cm}$ de profundidade, destorroadas e reviradas manualmente em caixas plásticas. Com uma pinça foi efetuada a captura da fauna edáfica e a identificação desses realizada a nível de grupos taxonômicos. Uma amostra de solo de cada camada citada anteriormente foi depositada sob funil com tela metálica de $5 \mathrm{~mm}$, coberta por outra tela de polietileno de $2 \mathrm{~mm}$, recebendo radiação luminosa (lâmpadas) por 7 dias consecutivos em uma estrutura denominada Funil de Berlese-Tullgren, para extração da meso fauna.

\section{Índices de avaliação da meso e macrofauna}

A comunidade da meso e macrofauna invertebrada edáfica foi avaliada por meio da abundância (número total). A significância estatística das diferenças observadas entre os 
locais onde foram recolhidas as amostras, bem como a influência das variações ambientais (estação do ano) sobre a fauna edáfica foram avaliadas pela análise da variância não paramétrica de Kruskal-Wallis. A similaridade entre as amotras nos diferentes tramentos (pastoreio continuo e PRV) e os estratos do solo (cobertura, 0 a 10 $\mathrm{cm}$ e de 10 a $20 \mathrm{~cm}$ ) foi analisada utilizando coeficiente de Sørensen. Para a diversidade de grupos funcionais se utilizou o índice de Shannon): o índice de diversidade de Shannon foi obtido pela relação $\left(\mathrm{H}=-\mathrm{Pi} \log\right.$ pi), em que: $\mathrm{Pi}=n \mathrm{ni}^{*} \mathrm{~N}^{-} 1$; onde ni é a densidade de cada grupo e N é o número total de grupos, conforme Odum (1983). A dominância de grupos taxonômicos foi analisada utilizando o índice de Berger-Parker, no qual: $\mathrm{d}=$ Nmax/N Índice de Dominância. Neste, Nmax é o número de indivíduos da espécie mais abundante, e N é o número total de indivíduos da comunidade (May, 1975).

\section{RESULTADOS E DISCUSSÃO}

\section{Abundancia da Fauna edáfica nos diferentes sistemas de pastejo}

A abundancia da macrofauna do solo variou em função da época de amostragem e do tratamento (Fig.1). A alta abundancia de insetos encontrados no inverno demonstram as boas condições no período estudado. A maior abundancia de insetos pode ter relação com a precipitação maior do que a média para o período. Outros fatores que podem ter contribuído para o aumento da abundância de insetos do inverno foram a quantidade e a qualidade das espécies forrageiras. No entanto, a abundancia da fauna edáfica considerando o sistema de pastoreio, continuo e o PRV no período de inverno, acumulando todos os estrados analisados, não foi significativamente diferente $(\mathrm{P}=0,57)$. No período de primavera, apesar de termos uma densidade mais baixa de insetos em relação ao inverno foi observada uma diferença significativa entre os dois sistemas de pastoreio analisados, no estrato de solo de 0 a $10 \mathrm{~cm}$ de profundidade, onde o grupo de insetos do PRV se apresentou em maior abundancia (estrato 0 a 10, $(\mathrm{P}=0,04)$. O deferimento do campo nativo implementado pela utilização do sistema PRV e as condições climatológicas da primavera, corroboram com um maior crescimento da pastagem nativa. Laossi et al (2008) sugerem que a densidade de fauna edáfica é afetada 
pela qualidade e pela quantidade de cobertura vegetal. Em um experimento realizado por Silva et al. (2008) no cerrado brasileiro, observou-se que minhocas responderam de forma positiva ao sistema integrado na fase de pastagem, provavelmente, pela maior densidade de raízes que as pastagens produzem e ao acúmulo de dejetos dos animais que melhoram a qualidade da matéria orgânica, o que também é observado no sistema PRV. Entre os estratos ouve diferença na abundancia de insetos, tanto no sistema de pastoreio continuo como no PRV. Como era esperado, nos estratos de 10 a $20 \mathrm{~cm}$ a abundancia foi menor, constituída fundamentalmente por formas larvárias dos insetos e alguns ácaros.

Figura 1. Comparação da abundância da fauna edáfica entre os estratos de solo, cobertura, 0 a $10 \mathrm{~cm}$ e 10 a $20 \mathrm{~cm}$ de profundidade, dos diferentes sistemas de pastoreio, continuo e PRV. As analises forma realizadas utilizando o teste de Kruskal-Wallis a $5 \%$.

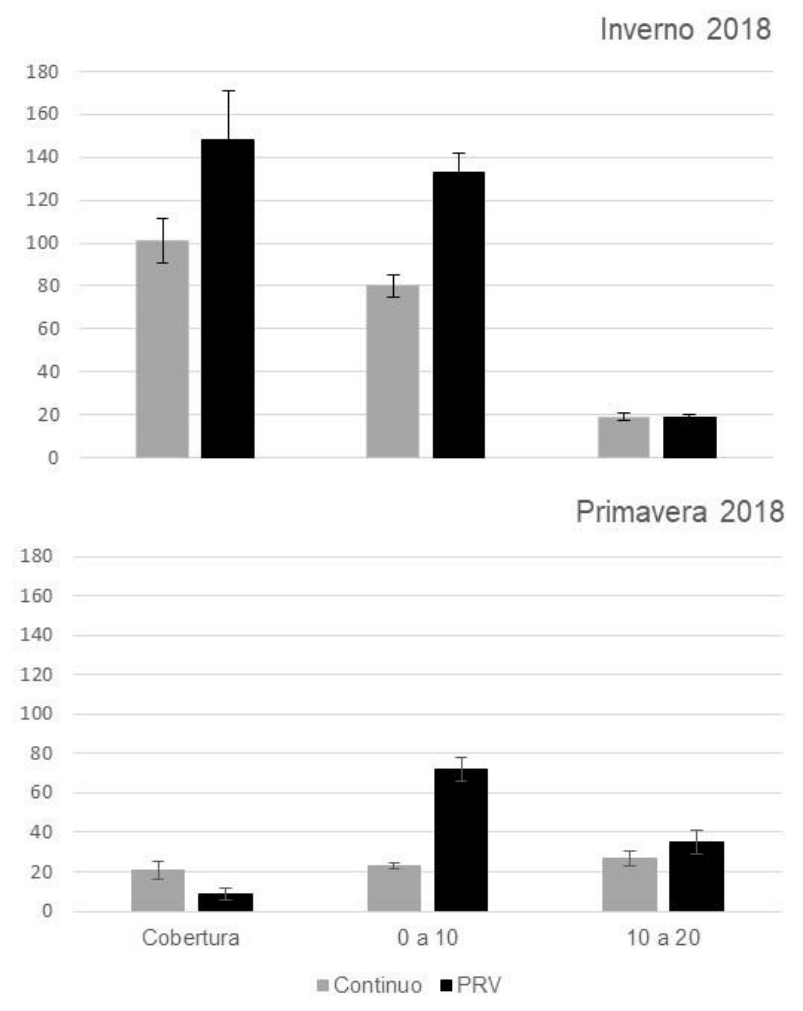

Análise da similaridade das espécies da fauna edáfica nos diferentes estratos de solo avaliados com diferentes sistemas de pastoreio

Para melhor entender o comportamento em relação aos estratos do solo e os 
tratamentos aplicados foi realizado o teste de similaridades das espécies que compõe a fauna edáfica (Fig. 2).

Figura 2. Dendrograma de Similaridades de acordo com o índice de Sørensen. Análise do Índice de Similaridade da fauna edáfica nos diferentes estratos de solo, nos períodos de inverno e primavera, com sistemas de pastoreio Continuo e PRV.

Inverno 2018

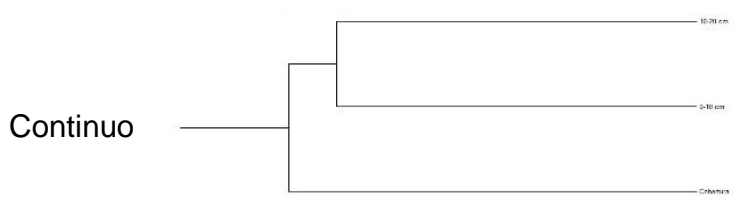

PRV

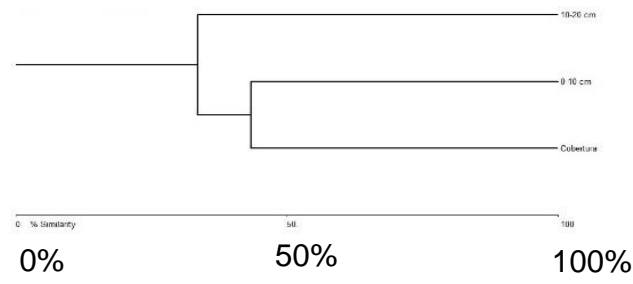

Primavera 2018

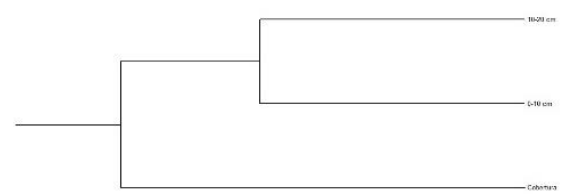

$0-10$

Cobertura
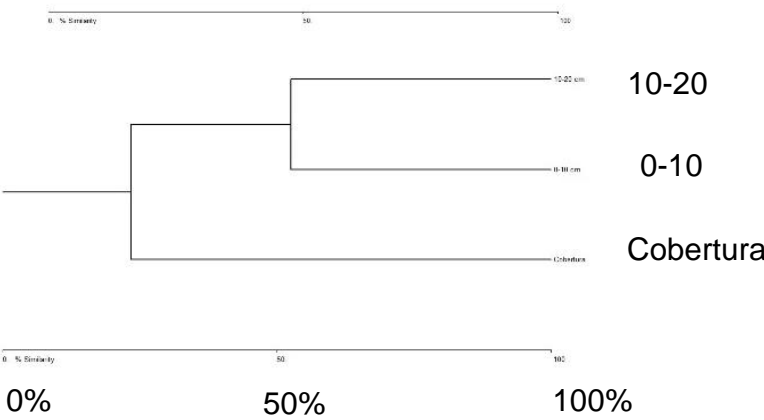

O coeficiente de similaridades de Sorensen indica que as áreas amostradas são distintas quanto a composição de insetos, levando em consideração a presença ou ausência destas. As espécies encontradas nos estratos 0 a $10 \mathrm{~cm}$ e de 10 a $20 \mathrm{~cm}$ são altamente similares em relação a estação do ano e aos sistemas de pastoreio analisados, com exceção do sistema PRV no inverno, onde as espécies da fauna edáfica foram mais similares na cobertura e nos estratos de 0 a $10 \mathrm{~cm}$. Santos et al. (2008) observaram diferença significativa nas médias populacionais de artrópodes, com a utilização de plantas de cobertura de inverno, em comparação ao sistema em pousio. Neste sentido, é possível que o sistema de pastoreio PRV, que permite o acúmulo de plantas, inclusive arbustivas nessa região, sirva de proteção do solo, afetando positivamente a comunidade da fauna do solo.

\section{Classificação da fauna edáfica de acordo com a sua importância funcional}


A fauna do solo ou edáfica está constituída por organismos que passam ao menos uma parte de sua vida sobre a superfície do solo nos troncos secos, sobre as folhas depositadas na superficie ou abaixo da superfície. Para viver no solo, os organismos vêm se adaptando ao longo dos anos a um ambiente compacto, com baixa concentração de oxigênio e luminosidade, baixa disponibilidade e qualidade de alimentos e flutuações microclimáticas (Lavelle et al, 1992). Considerando a maneira em que esses que grupos pertencentes a fauna edáfica forma se adaptando ao ambiente não e de se surpreender que executem múltiplas funções nos ecossistemas terrestres, variando desde organismos denominados de engenheiros do solo (geófagos), que ingerem solo (responsáveis pelaconcentração da matéria orgânica humificada); detritívoros, descomponedores e desintegradores, que se alimentam de material vegetal o animal em decomposição (necrófagos), aceleram os processo de incorporação da matéria orgânica no solo; os fitófagos e rizófagos, que se alimentam de plantas vivas (raíces o partes aéreas) e os depredadores, que se alimentam de outros organismos tanto de origem vegetal como animal (Brown et al. 2002). Como exemplo de Engenheiro do solo temos o grupo dos Anelídeos (minhocas), das Himenópteras Formicidae (formigas)e das Isopteras (cupins); dos Detritívoros temos os Coleópteros principalmente os da família Escarabeídea que incorporam os excrementos de mamíferos ao solo; os Colembolas, muito sensíveis a trocas do ambiente, principalmente a agentes poluidores (por isso servem como indicadores de qualidade de ambiente), e os ácaros da família Oribátida. Os ácaros são os microartrópodos mais abundantes no solo, contribuem com a decomposição da matéria vegetal, onde se destacam os Oribátidos, já que os Mesostigmatas e Prostigmatas tem menor importância nessa função; dos Fitofago, a maioria dos coleópteros inclusive suas larvas, larvas de lepidópteros e os ortópteros Acridídea (grilos); dos depredadores, dípteros, aracnídeos, ácaros (Prostigmatas), entro outros. A figura 3 indica a presença das espécies encontradas no presente estudo.

Figura 3. Grupos funcionais da fauna edáfica e sua distribuição nos sistemas de pastoreio, continuo e PRV, considerando as duas estacoes do ano analisadas, Inverno e Primavera. As analises forma realizadas utilizando o teste de Kruskal-Wallis a 5\%. 


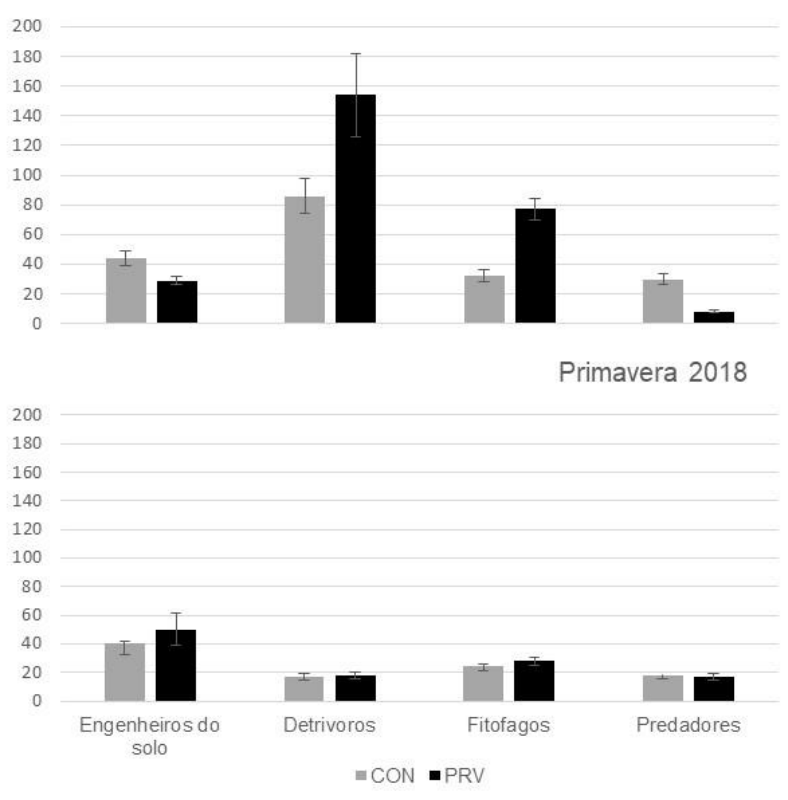

Como pode ser observado na figura 3 não houve diferença significativas entro os grupos, independentemente do tipo de pastoreio e da estação do ano. No entanto pode ser observada uma tendência positiva em relação a quantidade do grupo de detritívoros no sistema de pastoreio PRV no inverno. Esta tendência se deve ao fato que a presença dos ácaros Oribátidos foi 3 vezes maior no sistema PRV do que no Contínuo. Quando se compara diferentes famílias da fauna edáfica fica difícil saber o número necessário de cada uma para se estabelecer um índice de saúde do solo, considerando que o número entre famílias varia muito aumentando assim os desvios encontrados nas diferentes amostras.

\section{Diversidade da fauna edáfica}

O método de rarefação segundo Sanders (1968) propôs uma solução analítica para calcular todas as combinações possíveis de amostras de determinado tamanho para uma comunidade. O número esperado de espécies, baseado nas curvas de rarefação (Fig. 4), indica que a diversidade da fauna edáfica foi diferente no sistema de pastoreio PRV durante o período de inverno. Durante este período, o PRV propiciou que um maior número de espécies estivesse presente no solo. Com o índice de rarefação a tendência 
observada na abundância total de indivíduos no PRV de inverno se reflete no maior número de indivíduos, chegando ao máximo de indivíduos esperados nas amostras (Fig. 4).

Figura 4. Curva de rarefação por indivíduos para os diferentes sistemas de pastoreio (Contínuo e PRV) e as estações do ano (Inverno e Primavera).

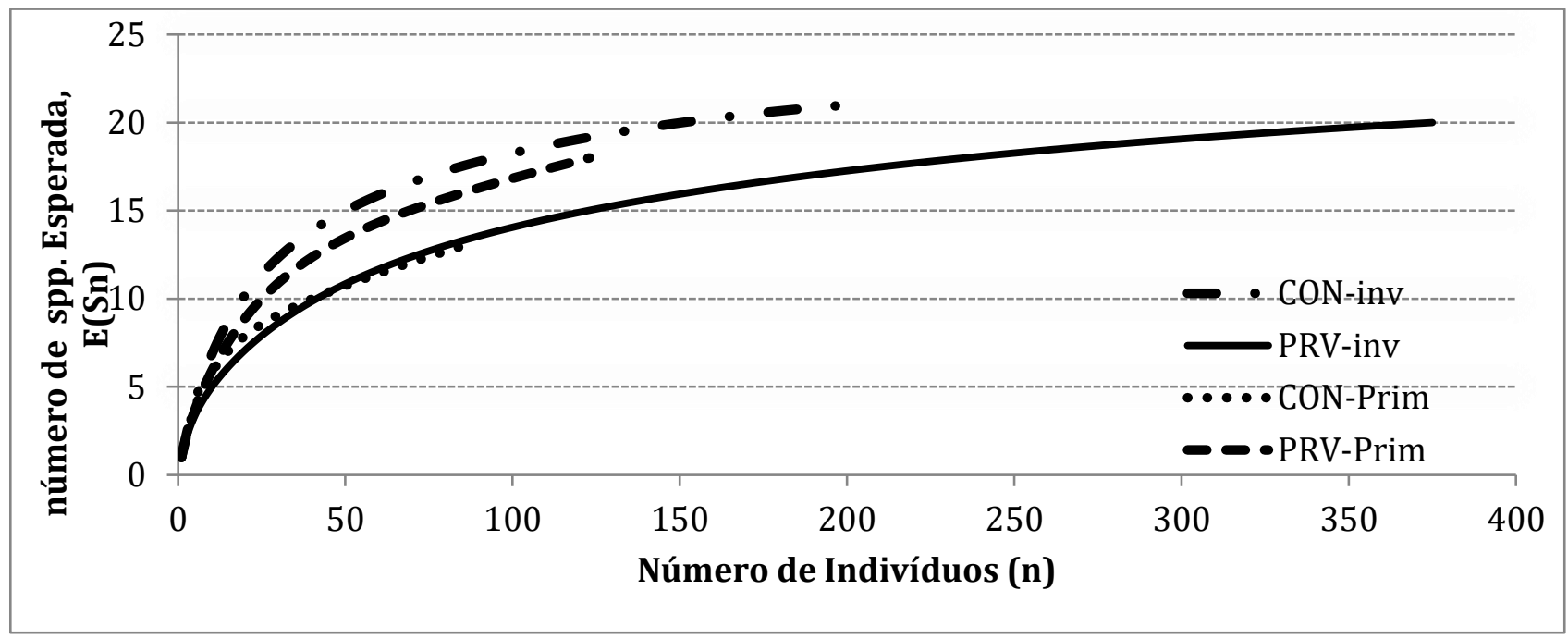

Através da análise gráfica verifica-se que no PRV de inverno, apesar do número expressivo de fauna encontrada nas amostras, a diversidade dos grupos taxonômicos diminui como mostram os índices de diversidade de Shannon $(\mathrm{H}=1,953)$, que foram menores que nos demais tratamentos, e de dominância de Berger-Parker $(0,3493)$, que ficou somente abaixo do tratamento PRV primavera (0,3821). Esses resultados estão de acordo com o que foi observado a campo, no PRV de inverno foi possível constatar alguns grupos dominaram as amostras do PRV de Inverno, como foi o caso dos ácaros oribatidos. Os ácaros oribátidos (Acari: Oribatida) apresentam grande potencial como bioindicadores já que são um dos grupos mais abundantes da mesofauna e possuem ciclos de vida relativamente longos (1 ou 2 anos), tem mobilidade restringida (o que limita a sua fuga de situações de estresse) e não conseguem aumentar sua densidade populacional em pouco tempo (Linden et al. 1994; Behan-Pelletier 1999). Isso nos indica que se os oribatidos estão presentes, como no caso do PRV de inverno, temos uma boa qualidade de solo em termos de conservação. 
Tabela 1. Índices de Diversidade comparando os sistemas de pastoreios, Continuo e PRV, nas estacoes do ano de Inverno e Primavera.

\begin{tabular}{lrrrr}
\hline & CON-inv & PRV- inv & $\begin{array}{r}\text { CON- } \\
\text { prim }\end{array}$ & PRV-prim \\
& & & & \\
\hline \hline Shannon_H & 2,58 & 1,953 & 2,097 & 2,205 \\
Berger- & 0,225 & 0,3493 & 0,2706 & 0,3821 \\
Parker & & & & \\
& & & & \\
\hline
\end{tabular}

\section{CONCLUSÃO}

O diferimento do campo nativo implementado pela utilização do sistema PRV e as condições climatológicas da primavera, corroboram com um crescimento da pastagem nativa o que interferiu positivamente na abundancia dos insetos encontrados estrados de solo de 0 a $10 \mathrm{~cm}$ de profundidade.

No sistema de pastoreio PRV durante o inverno foi observado uma mudança no comportamento dos insetos, em quanto ao estrato de solo e à similaridade desses insetos. O acúmulo de plantas no sistema PRV afetou positivamente a comunidade da fauna do solo nos estratos superficiais.

A presença dos ácaros Oribátidos foi três vezes maior no sistema PRV em relação ao pastoreio contínuo no período do inverno, indicando que esta metodologia de manejo do pastoreio beneficia a manutenção e proliferação da fauna edáfica.

\section{REFERÊNCIAS}

ANDERSON, J. M. Spatiotemporal effects of invertebrates on soil processes. Biology and Fertility of Soils. 6:3, 1988. 
ANDERSON, J.M., INGRAM, J.S.I. (1989) Tropical Soil Biology and Fertility: A

Handbook of Methods. CAB International, Wallingford.

BEHAN-PELLETIER, V. M. 1999. Oribatid mite biodiversity in agroecosystems: role for bioindication. Agriculture, Ecosystems and Environment, 74: 411- 423.

BERTON, C.T., RICHTER, M., PINHEIRO MACHADO FILHO, L. C. Referências Agroecológicas Pastoreio Racional Voisin. Cartilha PRV - Centro do Paraná de Agroecologia. Curitiba, 2011.

CRUZ, R.,BENAMU, M. A. Comparación de la edafofauna para cuatro usos de suelo. A4-608. In: Congreso Latinoamericano de Agroecología. Anais [...]. Mar del Plata, Argentina, 2015.

HUERTA-LWNGA， E., RODRIGUEZ， J., EVIA-CASTILLO， I., MONTEJOMENESES, E., CRUZ-MONDRAGON, M. de la, GARCIA-HERNANDEZ, R.. Relación entre la fertilidad del suelo y su población de macroinvertebrados. Terra Latinoamericana. 26: 71. 2008.

LAOSSI, K.R., BAROT, S, CARVALHO D., DESJARDINS, LAVELLE, P., MARTINS M., MITJA D., RENDEIRO A.C., ROUSSEAU G., SARRAZINI M., VELAZQUEZ E., GRIMALDI M. Effects of plant diversity on plant biomass production and soil macrofauna in Amazonian pastures. Pedobiol. 51: 397-407, 2008.

LINDEN, D., HENDRIX, F., COLEMAN, D. \& VAN VLIET, P. 1994. Fauna indicators of Soil Quality. In: Doran, J., Coleman, D., Bezdicek, D. \& Stewart, B. Anais [...] Soil Science Society of America - American Society of Agronomy, 35: 91-106. 
MARTIN, E.P., FEIJOO, A., PENA, J. Cuantificación de la macrofauna en un vertisol bajo diferentes sistemas de manejo en el Valle del Cauca, Colombia. Revista Suelos Ecuatoriales. 31:233, 2001.

MAY, R.M. 'Patterns of species abundance and diversity'. Ecology and Evolution of Communities, Cambridge, MA: Harvard University Press, pp. 81-120,1975.

MELADO, J. Manejo de Pastagem Ecológica - Um Conceito para o Terceiro

Milênio. 1. ed. Viçosa - MG: Aprenda Fácil Editora, 2000. v. 1. 224

ODUM, E.P. (1983) Basic Ecology. CBS College Publishing, New York.

SANTOS, G. G. et al. Macrofauna edáfica associada a plantas de cobertura em plantio direto em um Latossolo Vermelho do Cerrado. Pesquisa Agropecuária Brasileira, Brasília, DF, v. 43, n. 1, p. 115-122, 2008.

SCHIAVON, G. de A.; SCHUBERT, R. N.; PEREIRA, C. V.; LIMA, A. C. R. de; SCHIEDECK, G.; HOLZ, F. P.; FARIAS, E. S.; SCHWENGBER, J. E. Diversidade da fauna edáfica em duas estações do ano em sistema de produção de base ecológica de hortaliças no município de Morro Redondo - RS. In: CONGRESSO BRASILEIRO DE ZOOLOGIA. Anais [...] v. 29. p. 1567, 2012.

SILVA. C. A. Uso de Resíduos Orgânicos na agricultura. In: SANTOS, G. Fundamentos da matéria orgânica do solo: ecossistemas tropicais e subtropicais. 2. ed. Porto Alegre:Gênesis, 2008. 
VILLALOBOS, F.J., PULIDO, R.O., MORENO, C., PAVON, N.P., HERNANDENDEZ TREJO, H., BELL, J., MONTETIEL, S. Patrones de la macrofauna edáfica en un cultivo de Zea maiz durante la fase postcosecha en La Mancha, Veracruz, México. Acta Zoológica Mexicana. 80:16,2000.

ZERBINO, M.S. Evaluación de la densidad, biomasa y diversidad de la macrofauna del suelo en diferentes sistemas de producción. Tesis presentada en opción al título académico de Máster en Ciencias Ambientales. Facultad de Ciencias. Universidad de Montevideo, Uruguay. 92 p.,2005.

ZERBINO, M.S., ALTIER, N., MORON, A., RODRIGUEZ, C. Evaluación de la macrofauna del suelo en sistemas de producción en siembra directa y con pastoreo. Agrociencia, 12:44, 2008.

Recebido em: 20/10/2021

Aprovado em: 10/11/2021

Publicado em: 18/11/2021 